\title{
Estrategias de aprendizaje como una desigualdad educativa en el desempeño académico en tiempo de covid-19
}

\section{Learning strategies as an educational inequality in academic performance in the time of covid-19}

1 Helen Lizbeth Paucar Ojeda

Universidad Técnica de Ambato, Facultad de Ciencias de la Educación, Carrera de psicopedagogía. Ambato, Ecuador.

hpaucar4332@uta.edu.ec

2 Roberto Enrique Alvarado Quinto

Universidad Técnica de Ambato, Facultad de Ciencias de la Educación, Carrera de psicopedagogía. Ambato, Ecuador

re.alvarado@uta.edu.ec

3 Fredy Aguilar Rodríguez

Universidad Técnica de Ambato, Facultad de Ciencias sociales, Carrera de comunicación.

Ambato, Ecuador.

wf.aguilar@uta.edu.ec

4 Rodrigo Andrade Alban

iD https://orcid.org/0000-0002-8743-9

Universidad Técnica de Ambato, Facultad de Ciencias de la Educación, Carrera de

Psicopedagogía. Ambato, Ecuador

jr.andrade@uta.edu.ec

Artículo de Investigación Científica y Tecnológica

Enviado: 24/12/2021

Revisado: 29/12/2021

Aceptado: 12/01/2022

Publicado:08/03/2023

DOI: https://doi.org/10.33262/concienciadigital.v6i1.4.2056

Cítese. $\quad$ Paucar Ojeda, H. L., Alvarado Quinto, R. E., Aguilar Rodríguez, F., \& Andrade Alban R. (2023). Estrategias de aprendizaje como una desigualdad educativa en el desempeño académico en tiempo de covid-19. ConcienciaDigital, 6(1.4), 1113-1122. https://doi.org/10.33262/concienciadigital.v6i1.4.2056

CONCIENCIA DIGITAL, es una Revista Multidisciplinar, Trimestral, que se publicará en soporte electrónico tiene como misión contribuir a la formación de profesionales competentes con visión humanística y crítica que sean capaces de exponer sus resultados investigativos y científicos en la misma medida que se promueva mediante su intervención cambios positivos en la sociedad. https://concienciadigital.org

La revista es editada por la Editorial Ciencia Digital (Editorial de prestigio registrada en la Cámara Ecuatoriana de Libro con No de Afiliación 663) www.celibro.org.ec 
Palabras claves: educación, metas, aprendizaje, estrategias.

Keywords:

education, goals, learning, strategies.
Resumen

Las desigualdades educativas son el resultado de las diferencias sociales y económicas que se encuentran pasando las familias en tiempos de coronavirus, en la cual se puede palpitar que los estudiantes presentan varias carencias al no poseer todos los recursos necesarios para seguir aprendiendo mediante el uso de estrategias de aprendizajes que emplean y estas afectan directamente para que los estudiantes tengan un óptimo desempeño académico. Las metas académicas tienen una estrecha relación con el desempeño académico estas metas académicas pueden variar según su tipología, las metas de valoración social se puedo evidenciar en el grupo de los participantes que un porcentaje bajo ya que estos no les gusta realizar sus actividades académicas y si lo hacen es solo por buscar la aprobación de las demás personas y encajar en la sociedad de igual manera, las metas de aprendizaje tiene una influencia en cuanto a un mejor desempeño académico de los estudiantes obteniendo mayor habilidad en las materias de historia, lectura crítica y matemáticas.

\section{Abstract}

Educational inequalities are the result of the social and economic differences that families are going through in times of coronavirus, in which it can be felt that students have several deficiencies because they do not have all the necessary resources to continue learning using learning strategies that they employ, and these directly affect students to have an optimal academic performance. The academic goals have a close relationship with academic performance, these academic goals can vary according to their typology, the goals of social valuation can be evidenced in the group of participants that a low percentage since they do not like to perform their academic activities and if they do it is only to seek the approval of other people and fit into society in the same way, the learning goals have an influence in terms of better academic performance of students obtaining greater ability in the subjects of history, critical reading and mathematics. 


\section{Introducción}

En la actualidad las desigualdades educativas son el resultado de las diferencias sociales y económicas que se encuentran pasando las familias en tiempos de coronavirus, en la cual se puede palpitar que los estudiantes presentan varias carencias al no poseer todos los recursos necesarios para seguir aprendiendo mediante el uso de estrategias de aprendizajes que emplean y estas afectan directamente para que los estudiantes tengan un óptimo desempeño académico. De igual manera, las brechas digitales que existen ponen en riesgos a los estudiantes que viven en sectores alejados de la ciudad en dónde se puede encontrar todos los servicios educativos; de esta manera se ven afectados los estudiantes que requieren de tener un acompañamiento pedagógico para ir liberando los vacíos que se genera al no contar con todos recursos necesarios para obtener una educación de calidad y para todos aquellos que se encuentran pasando por las diferentes disparidades educativas en tiempos de Covid-19 (Lloyd, 2020).

Al realizar una reflexión sobre los tipos de desigualdades educativas se puede encontrar que existen las desigualdades legales, psicológicas, educativas, económicas y sociales dichas desigualdades generan consecuencias agravantes a cada ser humano en diferentes contextos de su vida cotidiana, pero en la desigualdad que se centra más este pequeño análisis es en la desigualdad educativa que se evidencia en tiempos de coronavirus es por ello que esta investigación tiene como objetivo identificar las estrategias de aprendizajes que poseen los estudiantes universitarios que afectan directamente en el desempeño académico en la realidad actual de confinamiento transformándose en una desigualdad educativa en tiempo de Covid-19.

Las estrategias de aprendizaje han venido evolucionando con el pasar de los años este procedimiento secuenciado no es más que un grupo de técnicas y estrategias que permite al estudiante adquirir, codificar y almacenar información atreves de conexiones sinápticas hasta la memoria de corto o largo plazo con el fin de que esta información sea utilizada en el ámbito académico, familiar y social (Visbal-Cadavid et al., 2017). Las estrategias de aprendizaje son un conector importante para seguir asimilando la información de forma consiste hasta que los estudiantes puedan ir plasmando la en su diario vivir mediante trabajos educativos, trabajos que se requiera evocar toda la información almacenada en su memoria ya que es de vital importancia despertar todos los conocimientos para llevar a cabo un buen desempeño académico en tiempos de cuarentena.

Las estrategias de aprendizajes se dividen en procesos de aprendizaje significativo, del recuerdo y aprendizaje memorístico:

- Aprendizaje significativo. - El aprendizaje significativo consta de dos tipos que son de elaboración y de organización cuyo objetivo es obtener un procesamiento 
simple, complejo en las cuales se utiliza habilidades o técnicas que son: Mapas conceptuales, parafraseo, resúmenes, organizadores y palabras claves.

- Aprendizaje del recuerdo. -La estrategia del recuerdo se basa en la evocación de la información y recuperación de esta en la cual emplea las técnicas de búsqueda directa en fuentes primarias.

- Aprendizaje memorístico. - El aprendizaje memorístico se enfoca en las estrategias de recirculación de la información la cual consiste en un repaso simple de la información apoyo de las técnicas de subrayar, copiar, resumir en fragmentos claros y concisos, de repetición acumulativa o simple.

Las estrategias de aprendizaje son importantes en el proceso educativo ya que esta nos permite resolver los problemas y generar una respuesta a las diferentes situaciones o problemas que se hallen los estudiantes en el proceso de aprendizaje de igual manera, las estrategias de aprendizajes le permite a los estudiantes reconocer el momento oportuno en el cual deben ser empleadas para cubrir las necesidades pedagógicas y a su vez ser compensadoras con un óptimo desempeño académico; las estrategias que los estudiante universitarios utilizan regularmente son las estrategias de adquisición mismas que les permite mantener el foco de atención para obtener conocimientos mediante el subrayado, de igual manera, se puedo encontrar que los estudiantes utilizan las estrategias de codificación de manera regular por medio de resúmenes u organizadores visuales que les permita tener la información a la mano. Asimismo, las estrategias de apoyo se encuentran siendo utilizadas en un porcentaje bajo lo que ocasiona que los estudiantes no puedan regular sus emociones ni habilidades para aprender y en ocasiones que no mantengan una conducta adecuada para generar un buen funcionamiento en su sistema cognitivo.

Los estudiantes en su proceso educativo tienden alcanzar metas para poder identificar su progreso, a dicho progreso nosotros lo llamaremos metas académicas las cuales se basan en diversos patrones como pueden ser creencias, afectos, sentimientos, atribuciones que rigen tanto las acciones como las intenciones del ser humano de igual manera, las metas académicas llevan consigo un propósito, motivación, intención y necesidad que tiene un estudiante para realizar tareas de forma autónoma. Por otra parte, se trata de que el aprendizaje del estudiante sea significativo y logre sus propósitos planteados en su vida (Moreno et al., 2019).

Las metas académicas tienen una estrecha relación con el desempeño académico estas metas académicas pueden variar según su tipología, las metas de valoración social se puedo evidenciar en el grupo de los participantes que un porcentaje bajo ya que estos no les gusta realizar sus actividades académicas y si lo hacen es solo por buscar la aprobación de las demás personas y encajar en la sociedad de igual manera, las metas de aprendizaje tiene una influencia en cuanto a un mejor desempeño académico de los estudiantes obteniendo mayor habilidad en las materias de historia, lectura crítica y matemáticas. Por 
último, las metas de logro en las cuales los estudiantes presentan un mejor desenvolvimiento en materias exactas como son lenguaje y comunicación, matemática y estadística (Rodríguez-Rodríguez \& Guzmán, 2018).

Las estrategias de aprendizaje a lo largo del tiempo han sido de gran importancia para que los estudiantes puedan adquirir todos los conocimientos básicos para su vida cotidiana es por ello que en este análisis de esta investigación se encontró los diferentes tipos de teorías de aprendizaje como son: La teoría de conductismo, cognitivismo, conectivismo y constructivismo mismas que son de importancia para que los estudiantes aprendan con diferentes estrategias empleadas por ellos mismos generando un óptimo desempeño académico y así mantengan sus altas expectativas en las metas que han se planteado a lo largo de su vida (Vega et al., 2019). El desempeño académico juega un rol importante en cuanto a las estrategias de aprendizajes pues estos están estrechamente vinculas ya que si los estudiantes logran aplicar las estrategias de aprendizaje de manera adecuada están les servirán para potenciar todo su conocimiento ocasionado que los resultados finales en su desempeño académico sean favorables. De igual manera, hay que aclarar que las metas académicas juegan un papel de gran importancia ya que por medio de estas los estudiantes se verán motivados para esforzarse cada día para alcanzar sus propósitos a lo largo de su vida estudiantil, profesional y familiar. Por consiguiente, estas metas académicas se han vistos afectadas por la pandemia del Covid-19 ya que los estudiantes han perdido de vista la importancia de aprender cada día ocasionando que las estrategias de aprendizaje que ellos emplean no estén generando el desempeño académico esperado tanto por ellos, como sus padres y profesores.

\section{Metodología}

En la presente investigación se utilizó el enfoque mixto, de campo, descriptivo, documental bibliográfico en el cual nos permitió recolectar toda la información que se ha detallado en la presente investigación de igual manera, se utilizó una población de 150 estudiantes entre hombres y mujeres de la Universidad Técnica de Ambato en la Facultad de Ciencias Humanas y de la Educación en la carrera de psicopedagogía con una muestra de tipo aleatoria-simple.

Para la evaluación de las estrategias de aprendizaje que poseen los estudiantes se utilizó la escala de estrategias de aprendizaje (ACRA) misma que consta de 119 ítems por los autores Román José y Gallego Sagrario mismo que permitió conocer el tipo de estrategias de aprendizaje que emplean los estudiantes en sus clases cotidianas de igual manera, para evaluar las metas de aprendizaje se utilizó la escala de Metas de Aprendizaje para Estudiantes Universitarios con 20 ítems de los autores Pérez María, Díaz Alejandro, Gonzáles Julio, Núñez José y Rosárico Pedro, el cual nos permitió conocer cuáles son sus expectativas para alcanzar sus metas en cuanto al logro académico y personal para obtener un buen desempeño académico en tiempos de Covid-19. Los datos se obtuvieron por 
medio de la encuesta aplicada por Google Forms misma que se procesaron por Microsoft office Excel.

\section{Resultados}

\section{Figura 1}

Escala de estrategias de aprendizaje (ACRA)

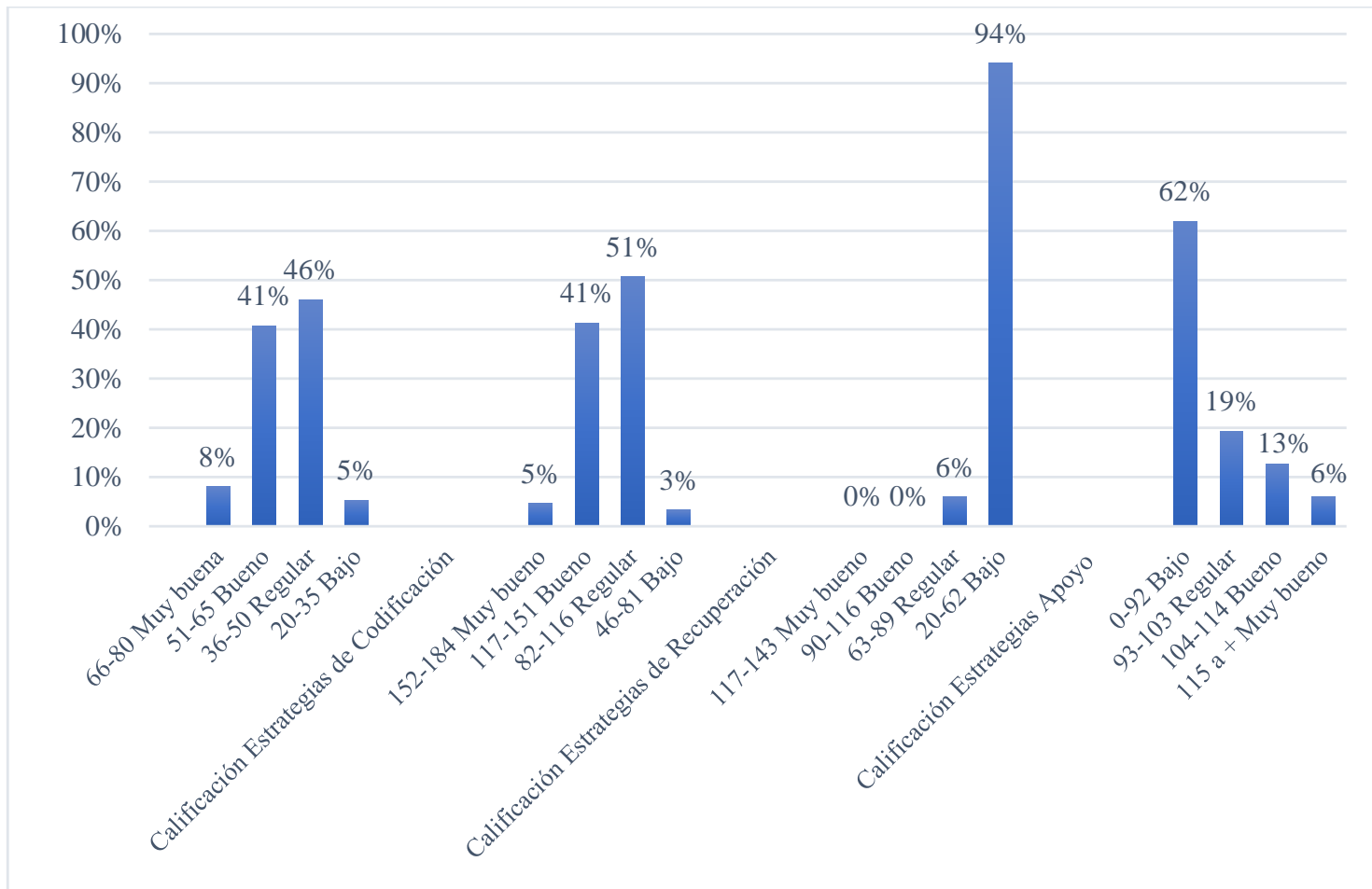

Nota: Distribución porcentual de los componentes de la escala ACRA. Fuente: Estudiantes de la Universidad Técnica de Ambato carrera de psicopedagogía.

Una vez aplicado la escala ACRA la cual nos define las estrategias desde los componentes en lo cual su primer componente referido a las estrategias de adquisición con un porcentaje de $94 \%$ dónde se puede evidenciar que los estudiantes no hacen un uso adecuado al momento de implementar este tipo de estrategias como son: la atención mismo que se implementa para adquirir nuevos conocimientos en las aulas de clases el cual sirve para retener información para después ser almacenada de forma correcta se debe implantar un repaso y subrayado para obtener un buen desempeño académico, como lo menciona Parada-Barrera et al., (2017), en su artículo denominado Dimensiones del aprendizaje y sus estrategias ACRA (adquisición, codificación,, recuperación y apoyo) utilizadas por estudiantes de la Lic. En biología de la Universidad de Guadalajara en la que menciona que los estudiantes presentan las estrategias de recuperación en un $41 \%$ en la que se puede interpretar que implementa de manera buena al momento de recuperar la información que se encuentra almacenada en la memoria de largo plazo para así ser 
utilizada en los momentos que considera oportuno el estudiante generando un buen desempeño académico.

\section{Figura 2}

Escala de mestas de estudio para estudiantes universitarios

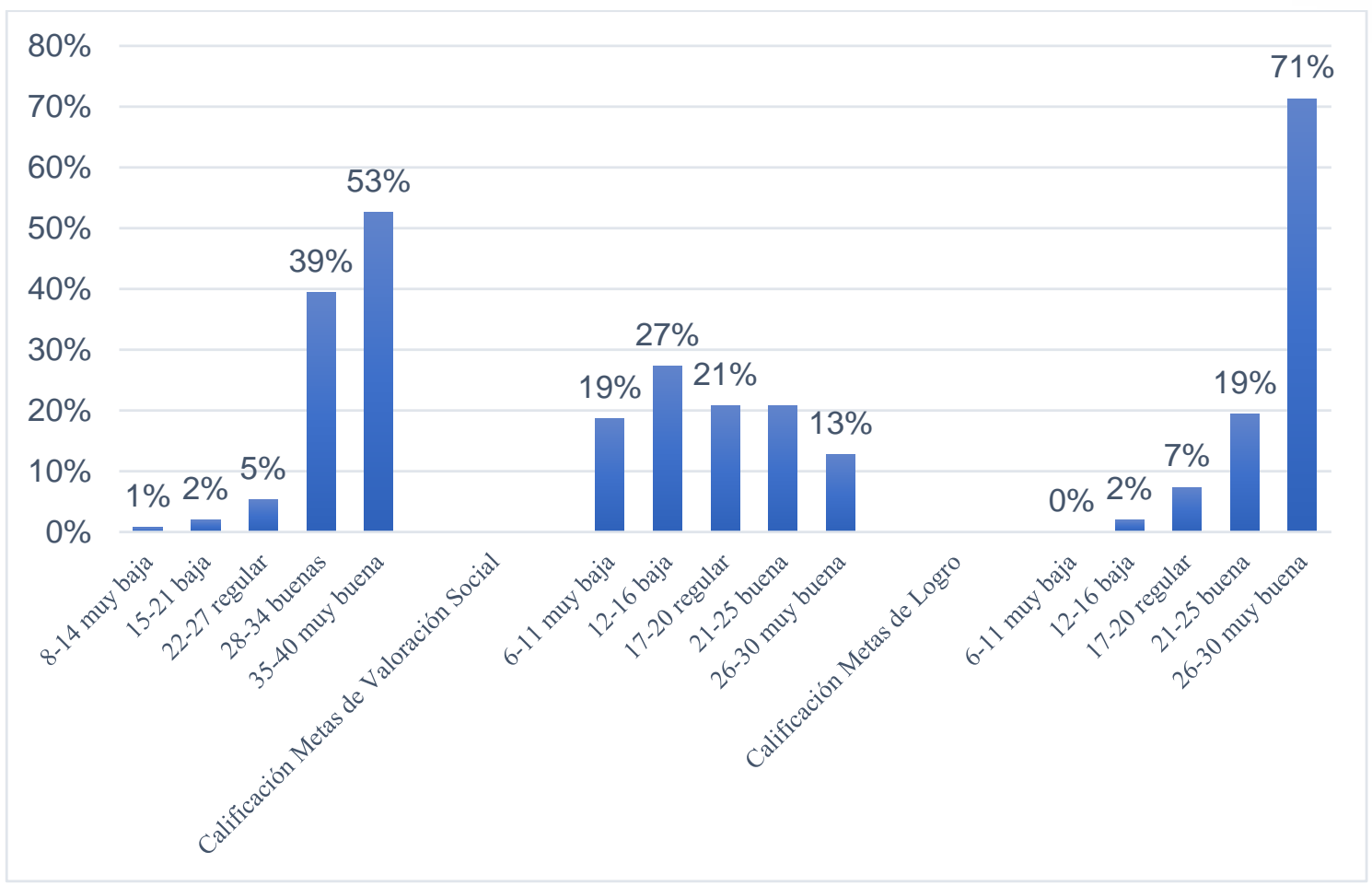

Nota: Distribución porcentual de los componentes de la escala de metas de estudios para estudiantes Universitarios. Fuente: Estudiantes de la Universidad Técnica de Ambato carrera de psicopedagogía.

Obtenido los resultados de la aplicación de la escala de metas de estudio para estudiantes Universitarios se puede evidenciar que existe un $71 \%$ en el componente de metas logro cual indica que los estudiantes se encuentran motivados para alcanzar buenos resultados en su desempeño académico por los logros que ellos desean alcanzar planteados en su vida académica. Ariza et al. (2020), hace referencia en su artículo denominado Asociación de metas y motivaciones académicas en relación con la variable sexo que existe un al porcentaje en el sexo de las personas refiriéndose que las personas del sexo masculino presentan más desarrolla el componente de las metas de aprendizaje ay que se enfocan más en la adquisición de nuevos conocimientos que le servirán a futuro más que obtener elogios o recompensar por sus progenitores o decentes.

\section{Conclusiones}

- Por medio del estudiado realizado sobre el tema: Las estrategias de aprendizajes son muy importantes y mantienen una estrecha relación con el cumplimiento del 
desempeño académico de los estudiantes, pero también debemos notar que en este tiempo de Covid-19 los estudiantes han cambiado las estrategias de aprendizaje que tenían razón por la cual también han cambiado el cumplimiento de su desempeño académico.

- El cambio de las estrategias de aprendizaje en tiempo de Covid-19 se ha producido debido a factores como: medios económicos, sociales, culturales, psicológicos entre otros; estos factores son percibidos de diferentes maneras por las personas ya que no todos tenemos una misma forma de aprendizaje y a esto sumamos que la educación utiliza esquemas de aprendizaje estandarizados olvidando los factores antes mencionados; a este olvido muchas veces involuntario lo llamamos como desigualdad educativa.

- En el caso de nuestro estudio se pudo notar en el tiempo de Covid-19, se evidenció que los estudiantes universitarios no implementan de manera adecuada las estrategias de aprendizaje como son: las estrategias de adquisición, codificación, recuperación y de apoyo en su hábito de estudio lo que implica que exista una desigualdad educativa ya que la mayor parte de los estudiantes no receptan la información de manera adecuado ocasionando que tengan un declive en su desempeño académico.

- De la misma forma, se evidencia que existe un alto índice de desmotivación al no ser reconocido los logros que alcanzan por el esfuerzo realizado en cada una de sus acciones siendo así una determinante para que exista una desigualdad educativa ya que todos los esfuerzos de los estudiantes no son reforzados ni valorados en la sociedad. Es por ello por lo que los estudiantes necesitan plantearse metas específicas a corto y largo plazo de manera que adquieran una satisfacción intrínseca de cada logro alcanzado.

\section{Referencias bibliográficas}

Ariza, C., Toncel, L., \& Blanchar, J. (2020). Asociación de metas y motivaciones académicas en relación con la variable sexo. Revista Boletín Redipe, 9(12), 286293.

Lloyd, M. (2020). Desigualdades educativas y la brecha digital en tiempos de COVID19. En H. Casanova Cardiel (Coord.), Educación y pandemia: una visión académica, (pp. 115-121).

Moreno, J., Chiecher, A., \& Paoloni, P. (2019). Los estudiantes universitarios y sus metas académicas. Implicancias en el logro y retraso de los estudios. Ciencia Docencia y Tecnología, 30(59 nov-abr). 
Parada-Barrera, G., Rimoldi-Rentería, M. D., \& Medina-Lerena, M. S. (2017). Dimensiones del aprendizaje y sus estrategias acra (adquisición, codificación, recuperación y apoyo) utilizadas por estudiantes. Revista de Pedagogía Critica, $1-2,1-8$.

Rodríguez-Rodríguez, D., \& Guzmán, R. (2018). Metas y rendimiento académicos en estudiantes con y sin riesgo en educación secundaria. Revista Mexicana de Psicología,

https://www.redalyc.org/articulo.oa?id=243059346003

Vega, N., Flores-Jiménez, R., Flores-Jiménez, I., Hurtado-Vega, B., \& RodríguezMartínez, J. (2019). Teorías del aprendizaje. XIKUA Boletín Científico de la Escuela Superior de Tlahuelilpan, 7(14), 51-53.

Visbal-Cadavid, D., Mendoza-Mendoza, A., \& Díaz Santana, S. (2017). Estrategias de aprendizaje en la educación superior. Sophia, 13(2), 70-81. https://www.redalyc.org/articulo.oa?id=413751844008

\section{¿Ciencia}


El artículo que se publica es de exclusiva responsabilidad de los autores y no necesariamente reflejan el pensamiento de la Revista Conciencia Digital.

\section{Ciencia}

El artículo queda en propiedad de la revista y, por tanto, su publicación parcial y/o total en otro medio tiene que ser autorizado por el director de la Revista Conciencia Digital.
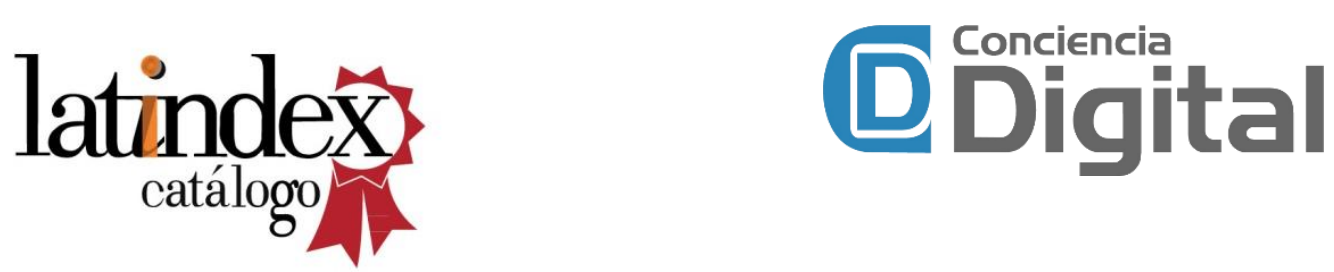

Indexaciones

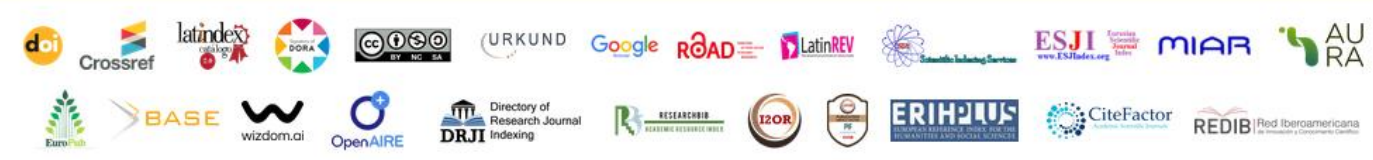

